\section{A case of sporadic periodic hypokalemic paralysis with atypical features: Recurrent differential right brachial weakness and cognitive dysfunction}

Sir,

We present a case of 28-year-old man admitted with an episode of fall due to weakness in all four limbs, with differential weakness of right upper limb that was associated with cognitive dysfunction. He had experienced multiple episodes of such attacks in the past 10 years. The relatives also noticed that after such an attack the patient remained confused for few hours. He did not give history of palpitations, hand tremors, or heat intolerance. His computed tomography scan of head and electroencephalogram suggested no abnormality. MRI of the brain was not done. A short review of systems was otherwise noncontributory. Neurological examination revealed the overall power in limbs was about $4 / 5$, but in the right upper limb it was found to be $1 / 5$. There was generalized hyporeflexia with a flexor plantars. His score on mini mental state examination (MMSE) was 21/30. The remainder of the physical examinations was noncontributory. Serum electrolyte: Potassium $1.7 \mathrm{mEq} / \mathrm{L}$ (normal range: $3.5-5.3 \mathrm{mEq} / \mathrm{L}$ ). He was investigated for renal loss of potassium which was normal. His thyroid stimulating hormone level (TSH) was reduced to $0.05 \mathrm{ng} / \mathrm{dL}$ (normal range: $0.45-4.5 \mathrm{ng} / \mathrm{dL}$ ) indicating a hyperthyroid state. He was given potassium supplementation after which his symptoms recovered completely along with improvement of cognitive functions without any residual weakness. His nerve conduction velocity (NCV) done during a nonparalyzed state was normal.
Periodic hypokalemic paralysis is often unrecognized when first encountered because of its relative rarity. ${ }^{[1]}$ Atypical presentations of severe hypokalemia may be in the form of total paralysis including respiratory, bulbar, and cranial musculature. ${ }^{[2]}$ Some patients complain of muscular weakness, especially of the lower extremities, while marked and generalized weakness of skeletal muscles is common with more severe potassium depletion, but they may present as weakness of specific group of muscles. Sudden deaths from respiratory failure and arrhythmia like ventricular tachycardia and fibrillation have been reported..$^{[3-5]}$ Our case was typical in presence of marked generalized weakness with differential weakness of right upper limb, reason of which cannot be explained. The sensations and level of consciousness are generally unaffected. Our patient had history of cognitive disturbance during the attack with hyporeflexia in all four limbs. Cognitive disturbances with hypokalemia have not been reported in literature. Both of the problems improved completely after potassium replacement which is a prerequisite for diagnosis of this disease.

\section{A. N. Joshi, A. P. Jain, A. D. Bhatt, S. Kumar Department of Medicine, Mahatma Gandhi Institute of Medical Sciences, Sewagram, Wardha, Maharashtra, India.} E-mail:dr.anjalijoshi@gmail.com

\section{References}

1. Stedwell RE, Allen KM, Binder LS. Hypokalemic paralyses: A review of the etiologies, pathophysiology, presentation, and therapy. Am J Emerg Med 1992;10:143-8.

2. Manoukian MA, Foote JA, Crapo LM. Clinical and metabolic features of thyrotoxic periodic paralysis in 24 episodes. Arch Intern Med 1999;159:601-6.

3. Chia BL, Lee KH, Cheah JS. Sino-atrial Wenckebach conduction in thyrotoxic periodic paralysis: A case report. Int J Cardiol 1995;47:285-9.

4. Wu CZ, Wu YK, Lin JD, Kuo SW. Thyrotoxic periodic paralysis complicated by acute hypercapnic respiratory failure and ventricular tachycardia. Thyroid 2008;18:1321-4.

5. Miyashita Y, Monden T, Yamamoto K, Matsumura M, Kawagoe N, Iwata C, et al. Ventricular fibrillation due to severe hypokalemia induced by steroid treatment in a patient with thyrotoxic periodic paralysis. Intern Med 2006;45:11-3.

Accepted on 25-02-2009 